\title{
Toward elucidating the mechanisms that regulate heterophylly
}

\author{
Hokuto Nakayama $^{1}$, Naomi Nakayama ${ }^{2}$, Akiko Nakamasu ${ }^{3}$, Neelima Sinha ${ }^{4}$, and Seisuke Kimura ${ }^{1}$ \\ ${ }^{1}$ Department of Bioresource and Environmental Sciences, Kyoto Sangyo University, \\ Motoyama, Kamigamo, Kita-Ku, Kyoto City 603-8555, Japan \\ ${ }^{2}$ Institute of Plant Sciences, University of Bern, Altenbergrain 21, CH-3013 Bern, Switzerland \\ ${ }^{3}$ Organization for the Strategic Coordination of Research and Intellectual Property, Meiji University, \\ 1-1-1 Higashimita, Tamaku, Kawasaki 214-8571, Japan \\ ${ }^{4}$ Section of Plant Biology, University of California at Davis, 1 Shields Avenue, Davis CA 95616, USA
}

Summary: The leaves of some plant species are able to change their morphology in response to environmental conditions. This phenomenon is termed heterophylly. Various aquatic plants exhibit drastic changes in leaf shape in response to submerged aquatic conditions. Heterophyllic variation ranges from mere modification of leaf width to drastic alteration in the outline of leaves and is interpreted as an adaptation to aquatic habitats. Although this phenomenon is widely observed among angiosperms, there is limited information on the regulation of heterophyllic switch in leaf development. Here, we have reviewed existing knowledge on leaf development and heterophylly and have introduced Neobeckia aquatica as an emerging model to elucidate the mechanisms underlying heterophylly.

Key words: Heterophylly, heteroblasty, leaf morphology, leaf development, environmental plasticity, evolution

\section{Introduction}

The leaves of plants show tremendous variation in size and shape, despite all leaf primordia initially developing as simple protrusions from the flanks of the shoot apical meristem (Tsukaya 2006). Aside from variation among species, some species show abrupt changes in leaf form in response to surrounding environmental conditions. This phenomenon is termed heterophylly, and it is generally defined as the variation in leaf form in a single plant in response to environmental conditions (Zotz et al. 2011). Heterophylly allows us to study not only the environmental plasticity of plant development but also leaf development and regulation of leaf form. However, there is limited information on the mechanisms that regulate heterophylly, probably due to the lack of an adequate model, despite numerous morphological studies that have been conducted on this subject (Arber 1920, Bell 2008, Fassett 1957).

A thorough understanding of leaf development is required to understand heterophylly. Many recent studies have contributed toward improving our knowledge of leaf development. In addition to understanding the structure and development of leaves, many genes involved in leaf morphogenesis have been isolated, and their networks have been studied in several model species (Blein et al. 2009, Ichihashi et al. 2011, Moon and Hake 2011,
Szakonyi et al. 2010, Tsukaya 2006). Moreover, research on the morphological diversification of leaves during the course of evolution is also in progress. In particular, molecular mechanisms, such as those responsible for the difference between simple and compound leaves and the difference in the complexity of leaf form among related species, have been examined in several model plants and their close relatives (Hay and Tsiantis 2006, Kimura et al. 2008, Piazza et al. 2010). Furthermore, the links between phytohormones and leaf development have been examined in detail (Bilsborough et al. 2011, Braybrook and Kuhlemeier 2010, Koenig et al. 2009, Shani et al. 2010, Umehara et al. 2008). Indeed, phytohormones, particularly abscisic acid (ABA) and ethylene, are critical for heterophyllous transformation in various species (Anderson 1978, Hsu et al. 2001, Kane and Albert 1987, Kuwabara et al. 2003). Thus, it appears that the foundation has been laid toward elucidating the mechanisms of heterophylly

To facilitate our investigation on heterophylly, we selected Neobeckia aquatica (Eaton) Greene as the model system. N. aquatica is an aquatic member of Brassicaceae and is closely related to the genera Rorippa and Cardamine (Les 1994). N. aquatica exhibits distinct leaf morphology, depending on the environmental conditions; it exhibits simple leaves in terrestrial environments and 
compound leaves in submerged aquatic environments (Fig. 1). Additionally, N. aquatica offers some great advantages as an experimental system such as size of plants, easy to maintain, and similarity to the Arabidopsis. Here, we have reviewed the background on heterophylly and introduced $N$. aquatica as a new model system for studying heterophylly.

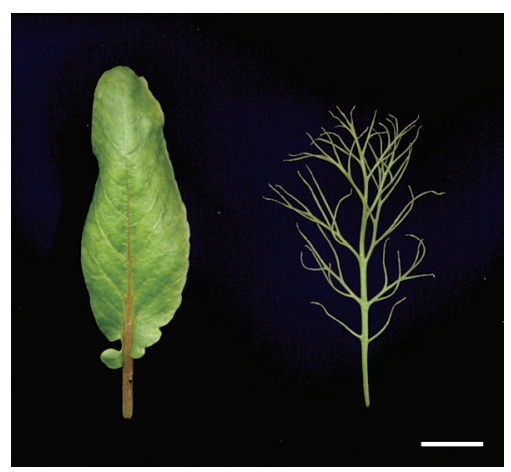

Figure 1 Comparison of the morphology of the simple and compound leaves of $N$. aquatica. Gross morphology of the terrestrial-type (left) and submerged-type (right) leaves of $N$. aquatica. Bar $=1 \mathrm{~cm}$.

\section{What is heterophylly?}

The definition of heterophylly has been subject to debate because of terminological confusion and lack of clear distinction from other similar developmental processes (Zotz et al. 2011). In this paper, we used Zotz's definition: environmentally induced switches between either of two (or more) possible leaf morphologies with typical example among amphibious plants, because the definition provides a clear description that is less likely to be misunderstood. In particular, we would like to clarify the difference between heterophylly and heteroblasty. Heteroblasty is a term that originally described conspicuous changes in leaves along the entire shoot during development (Goebel 1889, cited in Zotz et al. 2011). Hence, heteroblastic variations do not include morphological changes induced by any environmental stimuli. Thus, heterophylly is a distinct concept from heteroblasty.

Heterophylly is a phenomenon that is widely observed among diverse plant species, from ferns to angiosperms (Table 1). Many descriptive studies on the morphological change in leaves have been reported, with focus on aquatic plants (Anderson 1978, Bruni et al. 1996, Goliber and Feldman 1990, Hsu et al. 2001). Since the ancestors of land plants lived in aquatic habitats, aquatic plants are considered to have returned to aquatic environments from terrestrial habitats during evolution. Hence, heterophylly is thought to be one of several acquired phenotypes to enable life in submerged conditions. Morphological changes in leaf shape into narrow and/or compound form are generally thought to decrease the mechanical forces that act on the blade in underwater conditions. Therefore, heterophylly is generally regarded as an adaptation to water currents. However, the adaptive significance of heterophylly is still unclear because there are more factors to consider. For example, there are many other environmental changes associated with submerged conditions, such as light intensity and quality, availability of water and gases, and temperature. It is known that such factors may affect heterophylly. Thus, it is premature to assume that heterophylly is an adaptation to water currents alone. Furthermore, the so-called genetic costs of heterophylly should also be considered, as pointed out by Kleunen and Fischer (2005). The adaptive significance and fitness of heterophylly are 2 of the major questions that require consideration regarding leaf variation in response to submergence.

Unlike adaptive significance, the mechanisms of heterophylly have been studied in several species, with the results providing insights about how this phenomenon is regulated. In addition, research on leaf development and its molecular mechanisms has progressed remarkably in the last decade through the use of model species. These circumstances allow us to attempt to elucidate the mechanism of heterophylly. Below, we have summarized recent advances in the molecular mechanisms of leaf development as the basis for understanding heterophylly.

Table 1 Representative taxa of angiosperms known to contain heterophyllous species

\begin{tabular}{lll}
\hline & \multicolumn{1}{c}{ Family } & \multicolumn{1}{c}{ Genus } \\
\hline Basal angiosperm & Cabombaceae & Cabomba \\
& Nymphaeaceae & Nymphaea \\
Monocot & Aponogetonaceae & Aponogeton \\
& Alismataceae & Echinodorus, Sagittaria \\
& Pontederiaceae & Heteranthera \\
& Potamogetonaceae & Potamogeton \\
Eudicot & Brassicaceae & Nastatium, Neobeckia \\
& Haloragaceae & Myriophyllum, Proserpinaca \\
& Polygonaceae & Rumex \\
& Ranunculaceae & Ranunculus \\
& Onagraceae & Ludwigia
\end{tabular}




\section{Development of simple and compound leaves}

Leaf morphology is incredibly diverse. However, leaves can be roughly classified into 2 forms: simple, comprising a single blade, and compound, divided into multiple units termed leaflets. The developmental mechanisms of the both types of leaves have been studied in various model species. Here, we reviewed the molecular mechanisms of leaf development, with a focus on the following 2 model systems from Brassicaceae: the simple-leafed Arabidopsis thaliana and the compoundleafed Cardamine hirsuta. It is known that some aquatic species, including $N$. aquatica, switch between the 2 types of morphology in response to environmental conditions (Fig. 1). Thus, understanding the development of both leaf types is essential for elucidating the mechanisms that regulate heterophylly.

Leaf primordia initiate as bulges from the flanks of the shoot apical meristem (SAM). The SAM has a pluripotent state, which is characterized by the expression of class I KNOTTED1-LIKE HOMEOBOX $(K N O X)$ genes. The undifferentiated state of the SAM must be suppressed for leaf initiation to proceed. The expression of $K N O X$ is repressed in the presumptive leaf primordia. This downregulation is considered a landmark for the loss of meristem function and subsequent leaf initiation (Jackson et al. 1994, Long et al. 1996). Realtime imaging of the developing SAM indicated that localized auxin activity correlated with the region of KNOX downregulation, indicating that localized auxin accumulation is important for the downregulation of KNOX genes (Heisler et al. 2005). This repression is also mediated by the ASYMMETRIC LEAVES1/rough sheath 2/phantastica $(A R P)$ genes encoding MYB-domain containing proteins (Byrne et al. 2000, Timmermans et al. 1999, Waites et al. 1998). In Arabidopsis, the AS1 protein acts with the ASYMMETRIC LEAVES 2 (AS2) protein, which belongs to the AS2/LATERAL ORGAN BOUNDARIES (LOB) protein family, to repress $K N O X$ expression. The AS2 protein directly represses the transcription of BREVIPEDICELLUS $(B P)$, which is also known as KNAT1 (reviewed by Ueno and Springer 2011). Once repressed, $K N O X$ repression is maintained throughout leaf development in simple-leaved species such as Arabidopsis, and some reports suggest that the determinacy of leaves (i.e., lack of leaflet initiation) is associated with the repression of some genes, including KNOX (Jun et al. 2009, Kim et al. 2003, Scofield and Murray, 2006, Veit, 2009). After initiation, the primordia undergo certain processes, such as the establishment of adaxial and abaxial polarity and cell expansion (Braybrook and Kuhlemeier 2010, Tsukaya 2006). These processes increase organ size and determine final leaf shape. Subsequently, cells differentiate into specific tissues, such as vascular tissues and stomata.

However, the situation is different in compoundleaved species. For example, KNOX genes are expressed in the primordia in $C$. hirsuta. The expression is thought to promote a transient state of indeterminacy, during which leaflets form. In fact, in $C$. hirsute, the RNAi of the ortholog SHOOTMERISTEMLESS (ChSTM), which is a member of $K N O X$ genes, causes plants to develop leaves with fewer leaflets. Conversely, 35S::KN1-GR plants produce leaves with more leaflets (Hay and Tsiantis 2006). These results indicate that KNOX proteins are essential for leaflet formation in the compound leaves of C. hirsuta.

Recently, the specific mechanism of transcriptional regulation of $K N O X$ was reported. In the $5^{\prime}$ upstream region of $S T M$, the $\mathrm{K}$-box, which is a non-coding sequence, was identified to be important for the postinitiation repression of STM in leaf primordia (Uchida et al. 2007, Uchida et al. 2010). In fact, cis-regulatory diversification of STM has led to an alteration of the expression pattern of STM in leaf primordia and has contributed to differences in leaf form among related species (Piazza et al. 2010).

As described in this section, many studies implicate $K N O X$ genes in leaf formation and the diversification of leaf morphology, especially with respect to the shift between simple and compound leaves. In the future, understanding the environmental regulation of KNOX is likely to be a central component toward elucidating the mechanisms of heterophylly, in some heterophyllic plants. 


\section{Research on heterophylly}

A well-characterized aquatic plant that shows heterophylly is Ludwigia arcuata (Onagraceae). Developmental and physiological analyses of L. arcuata showed that the phytohormones $\mathrm{ABA}$ and ethylene are important for the change in leaf morphology. In $L$. arcuata, the application of exogenous ABA resulted in a terrestrial-type leaf form in submerged shoots, whereas ethylene induced submerged-type leaf form in terrestrial shoots. In addition, exogenous ABA did not affect the endogenous level of ethylene, whereas ethylene reduced the endogenous level of ABA (Kuwabara et al. 2003). Furthermore, ABA application was sufficient for the formation of the terrestrial-type leaf form in other heterophyllous aquatic plants (Hsu et al. 2001, Goliber and Feldman 1989, Kane and Albert 1987). Thus, the regulation of heterophylly induction via $\mathrm{ABA}$ may be a general trend in evolution. It is known that ABA plays a critical role in the response to cellular osmotic stress, including drought, for many plants. In the case of drought stress, $\mathrm{ABA}$ accumulates in vegetative tissues, resulting in the induction of ABA-responsive gene expression (Goda et al. 2008). In particular, ABA RESPONSIVE ELEMENT (ABRE) BINDING PROTEIN/ABRE BINDING FACTOR and G-box binding factors act in the transcriptional regulation of acclimation responses and tolerance of plants to drought (Hirayama and Shinozaki 2010). Moreover, the origin of the ABA signaling pathway is thought to be ancient, with the pathway being conserved in the green plant lineage (Takezawa et al. 2011). Therefore, the fact that ABA dependent pathways tend to be utilized in the heterophyllous regulation of aquatic plants is not surprising, even though heterophylly has evolved multiple times in distant plant species.

Meanwhile, the effect of ethylene on heterophylly remains relatively unclear, compared to that of ABA. In L. arcuata, ethylene treatment enhanced the elongation of epidermal cells; such cells are characteristically observed in the submerged-type leaves of aquatic plants (Sato et al. 2008). Cell elongation has also been observed in the stems of rice underwater. Genes for gibberellic acid (GA) are located downstream of that responsible for ethylene signaling in rice (Bailey-Serres and Voesenek 2010), with cell elongation being induced by GA treatment (Raskin and Kende 1984). However, the GA treatment of L. arcuata leaves did not induce cell elongation in terrestrial-type leaves (Kuwabara et al. 2001). These results indicate that the mechanism of heterophylly in L. arcuata is different from that in the rice stem, at least in the downstream components of the phytohormone signaling networks. Re-adaptation to aquatic habitats has occurred repeatedly during plant evolution, and it seems that the machinery to perceive environmental cues may be conserved, although the downstream response can be different.

\section{N. aquatica, an emerging model for studying heterophylly}

Although heterophylly has been studied in several plant species, it has been difficult to develop this research field further, because of shortage of experimental resources for the selected species. Thus, we sought a more versatile system to elucidate the mechanisms underlying heterophylly. Existing studies on leaf development have advanced by focusing on model species, such as Arabidopsis, tomato, or maize. However, these species do not show distinct heterophyllic transformation and, thus, are not completely suitable for research on this subject. To establish a new model system to study heterophylly, we selected $N$. aquatica (also known as lake cress), which is a member of Brassicaceae. N. aquatica shows dramatic heterophylly. For example, N. aquatica develops simple leaves (terrestrial-type) in air, whereas plants in submerged conditions develop compound leaves (submerged-type; Fig. 1).

$N$. aquatica is an excellent experimental system. First, it is easy to maintain. Indeed, the habitat of $N$. aquatica is quite diverse, including calm water; bays; and the muddy or rocky shores of lakes, streams, floodplains, and springs. Its growth requirements appear to be flexible. In addition, the plant is small in size (Fig. 2). Hence, N. aquatica can be easily grown in chambers, in the same way as Arabidopsis $\left(25^{\circ} \mathrm{C}\right.$, a short-day cycle); thus, it is possible to expose the plant to various environmental conditions 
in the laboratory. We are currently trying to identify what environmental cues induce heterophylly in $N$. aquatica by using a range of different controlled conditions in growth chambers. This research indicates that multiple environmental signals, besides submergence, might cause a heterophyllic switch. Moreover, we have collected several lines of $N$. aquatica that express different degrees of heterophyllous transformation in response to the environment.

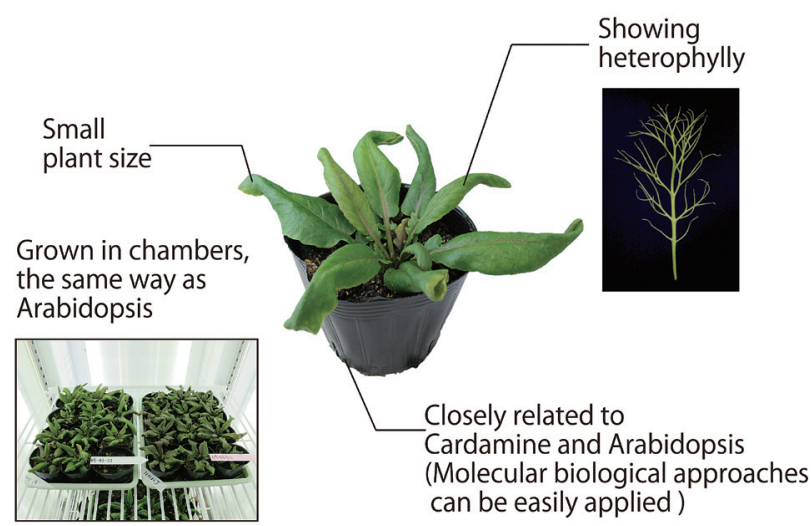

Figure 2 Schematic illustration of the advantages of $N$. aquatica as a model for studying heterophylly.

In addition, $N$. aquatica reproduces both sexually and asexually. Hence, regeneration can be used to maintain genetic homogeneity in N. aquatica. Moreover, because it also grows well in tissue culture, we are establishing an Agrobacterium-mediated transformation method for the functional analysis of genes involved in heterophyllic transformation.

Perhaps the greatest advantage of using $N$. aquatica is its similarity to the well-studied model systems of leaf development from the 2 genera Arabidopsis and Cardamine. Phylogenetic analysis by using the $r b c \mathrm{~L}$ gene suggested that Neobeckia is a monotypic genus and is close to Rorippa and Cardamine (Les 1994). Our molecular phylogenetic analysis, which was more extensive than that of Les (1994), also indicated a close relationship to Rorippa and Cardamine (Nakayama and Kimura, unpublished data). Therefore, it is likely that Neobeckia shares high similarity with respect to its genome and biological function with Cardamine, which in turn is related to Arabidopsis. The heterophyllic variations in Neobeckia leaves are likely to develop in a similar manner to that in the simple and compound leaves of Arabidopsis and Cardamine. Hence, the extensive knowledge accumulated using these 2 model species may help guide the research on Neobeckia. Most molecular biological procedures established for Arabidopsis or Cardamine are directly applicable to N. aquatica, without much modification. In addition, much of the information gathered from the genomic and biological system analyses of Arabidopsis is relevant to closely related plants such as Cardamine spp. and $N$. aquatica. Using this advantage, we have performed a next-generation sequencing-based genome-wide expression analysis by using the genomic information of Arabidopsis as a reference to identify the profile for differential expression by the 2 leaf forms. The results revealed molecular players that act on the heterophyllous transformation of $N$. aquatica and are currently being confirmed using quantitative RT-PCR and in situ hybridization.

The results of our experiments will help us understand the molecular framework underlying heterophylly. Plants are tremendously accommodating of their environment, at the level of a single plant, as indicated in the case of heterophylly. Environmental plasticity is essential for the survival of sessile plants. While we are just beginning to comprehend how heterophylly in N. aquatica is regulated, we believe that the knowledge gained from the studies on heterophylly will provide new insights about how plants respond to the environment and regulate shoot development.

\section{Acknowledgement}

This work was partially supported by a KAKENHI from the Ministry of Education, Culture, Sports, Science and Technology of Japan to S.K. (22870031); the Meiji University Global COE Program "Formation and Development of Mathematical Sciences Based on Modeling and Analysis to A.N. ; and a National Science Foundation grant to N.R.S. (IOS-0820854). 


\section{References}

Anderson, L. W. (1978) Abscisic acid induces formation of floating leaves in the heterophyllous aquatic angiosperm Potamogeton nodosus. Science 201: 1135-1138.

Arber, A. (1920) Water Plants. Cambridge University Press, London.

Bailey-Serres, J., and Voesenek, L. A. (2010) Life in the balance: a signaling network controlling survival of flooding. Curr Opin Plant Biol 13: 489-494.

Bell, A. D. (2008) Plant form: an illustrated guide to flowering plant morphology. Timber Press, Portland, Oregon.

Bilsborough, G. D., Runions, A., Barkoulas, M., Jenkins, H. W., Hasson, A., Galinha, C., Laufs, P., Hay, A., Prusinkiewicz, P., and Tsiantis, M. (2011) Model for the regulation of Arabidopsis thaliana leaf margin development. Proc Natl Acad Sci USA 108 (8): 3424-3429.

Blein, T., Hasson, A., and Laufs, P. (2009) Leaf development: what it needs to be complex. Curr Opin Plant Biol 13: 1-8.

Braybrook, S. A., and Kuhlemeier, C. (2010) How a plant builds leaves. Plant Cell 22: 1006-1018.

Bruni, N. C., Young, J. P., and Denglar N. G. (1996) Leaf developmental plasticity of Ranunculus flabellaris in response to terrestrial and submerged environments. Can J Bot 74: 823-837.

Byrne, M. E., Barley, R., Curtis, M., Arroyo, J. M., Dunham, M., Hudson, A., and Martienssen, R. A. (2000). Asymmetric leaves 1 mediates leaf patterning and stem cell function in $A$. thaliana. Nature 408: 967-971.

Fassett, N. C. (1957) A manual of Aquatic Plants. The University of Wisconsin Press, Madison, Wisconsin.

Goda, H., Sasaki, E., Akiyama, K., Maruyama-Nakashita, A., Nakabayashi, K., Li, W., Ogawa, M., Yamauchi, Y., Preston, J., Aoki, K., Kiba, T., Takatsuto, S., Fujioka, S., Asami, T., Nakano, T., Kato, H., Mizuno, T., Sakakibara, H., Yamaguchi, S., Nambara, E., Kamiya, Y., Takahashi, H., Hirai, M. Y., Sakurai, T., Shinozaki, K., Saito, K., Yoshida, S., and Shimada, Y. (2008) The AtGenExpress hormone and chemical treatment data set: experimental design, data evaluation, model data analysis and data access. Plant J 55: 526-42.

Goliber, T. E., and Feldman, L. J. (1989) Osmotic stress, endogenous abscisic acid and the control of leaf morphology in Hippuris vulgaris L. Plant Cell Environ 12: 163-171.

Goliber, T. E., and Feldman, L. J. (1990) Developmental analysis of leaf plasticity in the heterophyllous aquatic plant Hippuris vulgaris. Am J Bot 77: 399-412.

Hay, A., and Tsiantis, M. (2006) The genetic basis for differences in leaf form between Arabidopsis thaliana and its wild relative Cardamine hirsuta. Nat Genet 38: 942-947.

Heisler, M. G., Ohno, C., Das, P., Sieber, P., Ready, G. V., Long, J. A., and Meyerowitz, E. M. (2005) Patterns of auxin transport and gene expression during primordium development revealed by live imaging of the Arabidopsis inflorescence meristem. Curr Biol 15: 1899-1911.

Hirayama, T., and Shinozaki, K. (2010) Research on plant abiotic stress responses in the post-genome era: past, present and future. Plant J 61: 1041-52.

Hsu, T. C., Liu, H. C., Wang, J. S., Chen, R. W., Wang, Y. C., and Lin, B. L. (2001) Early genes responsive to abscisic acid during heterophyllus induction in Marsilea quadrifolia. Plant Mol Biol 47: 703-715.

Ichihashi, Y., Kawade, K., Usami, T., Horiguchi, G., Takahashi, T., and Tsukaya, H. (2011) Key Proliferative Activity in the Junction between the Leaf Blade and Leaf Petiole of Arabidopsis. Plant Physiol 157: 1151-1162.

Jackson, D., Veit, B., and Hake, S. (1994) Expression of maize KNOTTED1 related homeobox genes in the shoot apical meristem predicts patterns of morphogenesis in the vegetative shoot. Development 120: 405-413.

Jun, J. H., Ha, C. M., and Fletcher, J. C. (2009) BLADE$O N$-PETIOLE1 coordinates organ determinacy and axial polarity in arabidopsis by directly activating ASYMMETRIC LEAVES2. Plant Cell 22: 62-76.

Kane, M. E., and Albert, L. S. (1987) Abscisic acid induces aerial leaf morphology and vasculature in submerged Hippuris vulgaris L. Aquat Bot 28: 81-88.

Kim, M., Pham, T., Hamidi, A., McCormick, S., Kuzoff, R. K., and Sinha, N. (2003) Reduced leaf complexity in tomato wiry mutants suggests a role for PHAN and KNOX genes in generating compound leaves. Development 130: 4405-4415.

Kimura, S., Koenig, D., Kang, J., Yoong, F. Y., and Sinha, N. (2008) Natural variation in leaf morphology results from mutation of a novel KNOX gene. Curr Biol 18: 672-677.

Kleunen, V. M. and Fischer, M. (2005) Constraints on the evolution of adaptive phenotypic plasticity in plants. New Phytol 166: 49-60.

Kuwabara, A., Tsukaya, H., and Nagata, T. (2001) Identification of factors that cause heterophylly in Ludwigia arcuata (Onagraceae). Plant Biol 3: 98-105.

Kuwabara, A., Ikegami, K., Koshiba, T., and Nagata, T. (2003) Effects of ethylene and abscisic acid upon heterophylly in Ludwigia arcuata (Onagraceae). Planta 217: 880-887.

Koenig, D., Bayer, E., Kang, J., Kuhlemeier, C., and Sinha, N. (2009) Auxin patterns Solanum lycopersicum leaf morphogenesis. Development 136: 2997-3006.

Les, D. H. (1994) Molecular systematics and taxonomy of lake cress (Neobeckia aquatica; Brassicaceae), an imperiled aquatic mustard. Aquatic Bot 49: 149-165.

Long, J. A., Moan, E. I., Medford, J. I., and Barton, M. K. (1996). A member of the KNOTTED class of homeodomain proteins encoded by the SHOOTMERISTEMLESS gene of Arabidopsis. Nature 379: 66-69.

Moon, J., and Hake, S. (2011) How a leaf gets its shape. Curr Opin Plant Biol 14: 24-30.

Piazza, P., Bailey, C. D., Cartolano, M., Krieger, J., Cao, J., Ossowski, S., Schneeberger, K., He, F., Meaux, J. D., Hall, N., MacLeod, N., Filatov, D., Hay, A., and Tsiantis, M. (2010) Arabidopsis thaliana leaf form evolved via loss of KNOX expression in leaves in association with a selective sweep. Curr Biol 20: 2223-2228.

Raskin, I., and Kende, H. (1984) Role of gibberellin in the growth response of submerged deep water rice. Plant Physiol 76: 947-950. 
Sato, M., Tsutsumi, M., Ohtsubo, A., Nishii, K., Kuwabara, A., and Nagata, T. (2008) Temperature-dependent changes of cell shape during heterophyllous leaf formation in Ludwigia arcuata (Onagraceae). Planta 228: 27-36.

Scofield, S., and Murray, J. A. (2006). KNOX gene function in plant stem cell niches. Plant Mol Biol 60: 929-946.

Shani, E., Ben-Gera, H., Shleizer-Burko, S., Burko, Y., Weiss, D., and Ori, N. (2010) Cytokinin regulates compound leaf development in Tomato. Plant Cell 22: 3206-3217.

Szakonyi, D., Moschopoulos, A., and Byrne, M. E. (2010) Perspectives on leaf dorsoventral polarity. J Plant Res 123 : 281-290.

Takezawa, D., Komatsu, K., and Sakata, Y. (2011) ABA in bryophytes: how a universal growth regulator in life became a plant hormone? J Plant Res 124: 437-453.

Timmermans, M. C. P., Hudson, A., Becraft, P. W., and Nelson, T. (1999). ROUGH SHEATH2: A myb protein that represses knox homeobox genes in maize lateral organ primordia. Science 284: 151-153.

Tsukaya, H. (2006) Mechanism of leaf-shape determination. Annu Rev Plant Biol 57: 477-496.

Uchida, N., Townsley, B., Chung, K. H., and Sinha, N. (2007) Regulation of SHOOT MERISTEMLESS genes via an upstream-conserved noncoding sequence coordinates leaf development. Proc Natl Acad Sci USA 104: 15953-15958.

Uchida, N., Kimura, S., Koenig, D., and Sinha, N. (2010) Coordination of leaf development via regulation of KNOXI genes. J Plant Res 123: 7-14.

Ueno, Y., and Springer, P. (2011) Molecular genetic studies on ASYMMETRIC LEAVES2 (AS2) of Arabidopsis: Insight into the function of the AS2 protein. Plant Morphology 23: 7180 .

Umehara M., Hanada A., Yoshida S., Akiyama K., Arite., T, Takeda-Kamiya N., Magome H., Kamiya Y., Shirasu K., Yoneyama K., Kyozuka J., and Yamaguchi S. (2008) Inhibition of shoot branching by new terpenoid plant hormones. Nature 455: 195-200.

Veit, B. (2009). Hormone mediated regulation of the shoot apical meristem. Plant Mol Biol 69: 397-408.

Waites, R., Selvadurai, H. R., Oliver, I. R., and Hudson, A. (1998) The PHANTASTICA gene encodes a MYB transcription factor involved in growth and dorsoventrality of lateral organs in Antirrhinum. Cell 93: 779-789.

Zotz, G., Wilhelm, K., and Becker, A. (2011) Heteroblasty-A Review. Bot Rev 77:109-151.

Received: 27 January 2012 